\title{
El principio de proporcionalidad en el derecho de acceso a la información pública: usos y potencialidades para mitigar la discrecionalidad estatal al invocar una excepción legal. Una mirada desde el derecho argentino y la teoría de Robert Alexy
}

The Principle of Proportionality in the Right of Access to Public Information: Uses and Potentialities to Mitigate State Discretion when Invoking a Legal Exception. An Analysis from Argentine Law and Robert Alexy's Theory

Fátima LóPEZ PoletTI ${ }^{1}$

Resumen: El principio de proporcionalidad es un procedimiento de aplicación de normas sobre derechos fundamentales. Si bien nació en Alemania, hoy en día y gracias a la contribución de Robert Alexy, migró a otros sistemas jurídicos. Generalmente, su empleo se aborda desde la perspectiva judicial; es decir, como un mecanismo de control de constitucionalidad. Este trabajo aborda el principio de proporcionalidad desde otro enfoque, pues mi intención consiste en evaluar su eficacia en el ámbito particular del derecho de acceso a la información pública $\mathrm{y}$, por lo tanto, aplicable en el marco de las

\footnotetext{
${ }^{1}$ Abogada (UCA). Maestranda en Derecho Constitucional y Derechos Humanos (UP). Diplomada en Argumentación Jurídica, Interpretación Constitucional y Valor del Precedente (Observatorio de Abogados). Docente de Derecho Constitucional en la Facultad de Derecho de la Universidad Católica Argentina.
} 
decisiones adoptadas por el poder político. De este modo y dado que para Alexy la concepción sobre los derechos fundamentales como requisitos de optimización es indisociable del principio de proporcionalidad, analizaré cada uno de sus submandatos (idoneidad, necesidad y proporcionalidad en sentido estricto) teniendo en cuenta tal caracterización. En particular, enfatizaré en el "principalismo" (reconocimiento de principios y valores en las constituciones) como un elemento determinante de la ponderación. Finalmente, concluiré que el principio de proporcionalidad presenta una estructura argumentativa adecuada para fundamentar las excepciones al derecho de acceso a la información pública que, a la luz del principio de máxima divulgación, deben ser interpretadas restrictivamente.

Palabras claves: Principio de proporcionalidad, Derechos Humanos, Derecho de Acceso a la Información Pública, Argumentación constitucional

Abstract: The principle of proportionality is a procedure for the application of rules on fundamental rights. Although he was born in Germany, today and thanks to the contribution of Robert Alexy, it migrated to other legal systems. Generally, their implementation is approached from the judicial perspective; that is, as a mechanism of constitutional review. This paper addresses the principle of proportionality from another perspective, since my intention is to evaluate its effectiveness in the field of the right of access to public information and, therefore, applicable within the framework of decisions taken by political power. In this way, and given that for Alexy, the conception of fundamental rights as requirements for optimization is inseparable from the principle of proportionality. I will analyze each of its sub-mandates (suitability, necessity, and proportionality in a narrow sense) taking into account such characterization. I will emphasize "principalism" (recognition of principles and values in constitutions) as a determining element of the examination. Finally, I will conclude that the principle of proportionality presents an adequate argumentative structure to 
support the exceptions to the right of access to public information that, considering the principle of maximum disclosure, must be interpreted restrictively.

Keywords: Principle of proportionality, Human Rights, Right of Access to Public Information, Constitutional Reasoning

Recibido: 22.10.2021 Aceptado: 16.12.2021

Sumario

\section{Introducción}

\section{Principio de proporcionalidad}

a. La teoría de los principios y el principio de proporcionalidad

b. La estructura del principio de proporcionalidad: Ponderación. Necesidad de justificación racional

\section{El derecho de acceso a la información pública}

a. Caracterización

b. El principio de proporcionalidad en el derecho de acceso a la información pública

\section{Conclusión}

\section{Introducción}

Desde el año 2017 hasta el 2020, el fuero Contencioso Administrativo y Tributario de la Ciudad Autónoma de Buenos Aires recibió 188 amparos por acceso a la información pública, ${ }^{2}$ de los

\footnotetext{
${ }^{2}$ La cantidad de amparos por acceso a la información pública obedece al filtro "(incluye ley 104 y ambiental)" aplicado en la búsqueda del Sistema de Consulta Pública EJE (https://eje.juscaba.gob.ar/iol-ui/p/inicio). Sin embargo, se advierte que el número volcado no refleja la cantidad real de acciones promovidas por solicitantes que ven
} 
cuales sólo 101 cuentan con sentencia firme. De esos 101 casos, 45 son condenas al gobierno. Lo hasta aquí expuesto cobra especial relevancia si se tiene en cuenta que, de esos 45 expedientes, en 23 se invocó alguna excepción al derecho de información. Como puede apreciarse, los datos indican cierto déficit por parte de los sujetos obligados a la hora de justificar la limitación del derecho en cuestión en miras a la protección de otro al que, en el caso concreto, se le atribuye mayor peso. Mi punto, entonces, consiste en demostrar que ese déficit es argumentativo. En la práctica jurídica actual, la argumentación jurídica es central; en particular, cuando existe una colisión entre derechos. En lo que aquí interesa, dicha inteligencia adquiere mayor relevancia porque las derrotas ${ }^{3}$ generan severos perjuicios a nivel institucional; pues, no solo redundan en costos políticos -implican la pérdida de confianza de la ciudadanía sobre sus gobernantes porque se ven impedidos de ejercitar el control democrático de la gestión estatal—, sino también económicos —ya que las condenas cuestan dinero-.

Si bien es cierto que la argumentación jurídica es crucial para paliar este tipo de problemáticas, no es menos cierto que existe una gran variedad de técnicas aplicables a ese propósito. De hecho, en el ámbito del acceso a la información se han formulado diversas teorías que, en última instancia, intentan brindar respuesta a ese conflicto. Entre ellas se destacan: el test del daño y del interés público. Ambas tienen algo en común: suponen un mecanismo de "balanceo" entre dos derechos opuestos que, a decir verdad, recogen cada una de las tres etapas de la ponderación. Esto es razonable si se considera, por un lado, que la Corte Interamericana de Derechos Humanos (en

insatisfechos sus pedidos de información; pues se ha observado que, en algunos supuestos, la caratulación de estos casos se efectúa como amparos genéricos, habeas data o amparos por mora.

${ }^{3}$ En este trabajo se entiende por "derrota" a todas aquellas causas judiciales en las que el Gobierno sea condenado no solo a brindar la información requerida en vía administrativa mediante una solicitud de información sino también a asumir las costas del proceso. 
adelante, "Corte IDH") se expidió sobre el derecho de acceso a la información y erigió al principio de proporcionalidad como un mecanismo adecuado para fundamentar la procedencia de las excepciones a él; y, por el otro, que la ponderación forma parte del principio de proporcionalidad. En efecto, este trabajo se propone evaluar si su aplicación es conveniente para brindar razones a favor $o$ en contra de derechos contrapuestos que sean susceptibles de control posterior. Para ello, se describirá en qué consiste dicho principio y se evaluará si su aplicación puede resultar útil para justificar la reserva al acceso a la información. Cabe aclarar que si bien numerosos tribunales han optado por emplear tal mecanismo (por ejemplo, el Tribunal Constitucional Federal Alemán, la Suprema Corte de Justicia de la Nación en México, el Tribunal Europeo de Derechos Humanos, la Corte Interamericana de Derechos Humanos, entre otros), este trabajo tomará como punto de partida la teoría de Robert Alexy dado que, a nivel teórico, fue quien estudió con mayor sofisticación su estructura.

La postura aquí defendida será aquella en virtud de la cual la proporcionalidad, en tanto estructura argumentativa, es capaz de clarificar el camino del pensamiento desandado por la autoridad al momento de restringir un derecho fundamental. En consecuencia, aunque no pueda eliminar la discrecionalidad de las decisiones, es una herramienta útil a los efectos de controlar si ese razonamiento fue correcto o no.

\section{Principio de proporcionalidad}

Para Robert Alexy existe una relación entre la teoría de los derechos y la teoría del razonamiento jurídico que se consolida a partir del discurso racional, entendiendo por tal a un procedimiento para probar y fundamentar enunciados normativos y valorativos por medio de argumentos. Esto significa que: (i) la teoría del discurso racional es necesaria para proteger derechos fundamentales que, como tienen la estructura de principios, otorgan derechos prima facie 
(teoría de los principios); (ii) dichos derechos, para ser operativos, exigen la aplicación de un procedimiento ponderativo, es decir, del principio de proporcionalidad con sus tres subreglas: idoneidad, necesidad y proporcionalidad en sentido estricto (teoría del razonamiento jurídico); y, (iii) finalmente, para que la solución propiciada por el principio de proporcionalidad sea racional se deben brindar argumentos suficientes para justificarla y, eventualmente, controlar su legitimidad (teoría del discurso racional).

A continuación, se detalla el esquema trazado en el párrafo anterior.

\section{a. La teoría de los principios y el principio de proporcionalidad}

Los derechos fundamentales, en tanto preceptos constitucionales cargados de un alto grado de abstracción, generalidad e indeterminación, suelen ser catalogados como principios. En el esquema conceptual adoptado por Alexy, estos -a diferencia de las reglas- son entendidos como normas que ordenan que algo se cumpla en la mayor medida posible según las posibilidades fácticas y jurídicas. Las posibilidades fácticas dependen de cursos de acción alternativos; mientras que, las posibilidades jurídicas están dadas por la eventual existencia de reglas y principios opuestos. Los principios, entonces, son mandatos de optimización porque pueden ser cumplidos en distintos grados y son susceptibles de ponderación. Ante una eventual colisión, uno de ellos debe ceder frente al otro. Esto no implica que el principio desplazado sea inválido; sino que "bajo ciertas circunstancias uno de los principios precede al otro. Bajo otras circunstancias, la cuestión de precedencia puede ser solucionada de manera inversa” (Alexy, 1985, p. 89).

De lo expuesto se desprende que en tanto los principios son normas que tienen la misma jerarquía formal, no es posible establecer una precedencia absoluta entre ellos. Por eso, Alexy sostiene que para solucionar colisiones entre principios forzosamente han de ponderarse los intereses contrapuestos. De este modo, se trata de 
"establecer cuál de los intereses, abstractamente del mismo rango, posee mayor peso en el caso concreto" (1985, p. 90). La ponderación, entonces, es el procedimiento por medio del cual se establece una relación de preferencia condicionada entre principios.

La mentada relación reviste fundamental importancia porque de ella es posible derivar una regla, en cuya estructura se aprecia un supuesto de hecho y una consecuencia jurídica. En el primero se enuncian las condiciones en las que un principio precede a otro; mientras que, en la segunda, se determina la acción exigida por el principio prevalente. Lo hasta aquí descripto puede sintetizarse en una ley -la ley de colisión-, que se formula en los siguientes términos: "Las condiciones bajo las cuales un principio precede a otro constituyen el supuesto de hecho de una regla que expresa la consecuencia jurídica del principio precedente" (Alexy, 1985, p. 94).

Ahora bien, la decisión que resuelve cuál es el principio que precede a otro presupone la existencia de una estructura argumentativa que la justifique racionalmente. De acuerdo con la teoría de Alexy, eso es posible gracias al principio de proporcionalidad. Este se encuentra conformado por tres submandatos: el de idoneidad, el de necesidad y el de proporcionalidad en sentido estricto. Todos ellos expresan la idea de optimización; en consecuencia, interpretar los derechos fundamentales de acuerdo con el principio de proporcionalidad es tratarlos como mandatos de optimización. Los subprincipios de idoneidad y necesidad conciernen a la optimización relacionada con aquello que es fácticamente posible. En ellos la ponderación no juega ningún papel; pues, solo se trata de impedir ciertas intervenciones en los derechos fundamentales que sean evitables sin costo para otros principios. Es decir, expresan la idea del óptimo de Pareto — no existe un posible cambio que beneficie a una persona sin perjudicar a otra(Alexy, 2008, p. 15). Por su lado, el principio de proporcionalidad en sentido estricto está enfocado en la optimización de las posibilidades jurídicas. Por eso, ese es el campo de la ponderación. 
De lo expuesto se sigue que, para Alexy, existe una conexión conceptual entre su caracterización de los derechos fundamentales y el principio de proporcionalidad. Dicha relación se explica en el sentido de que su empleo posibilita el traspaso de un derecho prima facie a un derecho definitivo. En efecto, los derechos prima facie que tienen la estructura de principios-, constituyen mandatos de optimización que, para ser operativos, requieren la aplicación del principio de proporcionalidad, específicamente de la ponderación que es exigida por la ley de colisión. De ella se deriva un derecho definitivo, que tiene la estructura de una regla. Por consiguiente, los derechos prima facie exigen forzosamente el uso del principio de proporcionalidad para derivar derechos definitivos que prescriban una solución (consecuencia jurídica de la regla) para el caso concreto.

\section{b. La estructura del principio de proporcionalidad: Ponderación. Necesidad de justificación racional}

Dada la relevancia atribuida por Alexy al principio de proporcionalidad, se vuelve imperioso examinar cada uno de sus submandatos: idoneidad, necesidad y proporcionalidad en sentido estricto. Pues bien, el examen de idoneidad supone una relación de medio a fin; eso se debe a que exige que el medio escogido sea susceptible de alcanzar el objetivo propuesto. Tal es así que, si el medio no es adecuado para promover la satisfacción de un principio y, al mismo tiempo, afecta a un segundo principio, la adopción de ese medio queda prohibida en virtud de las posibilidades fácticas exigidas (Caminos, 2014, pp. 56 y 57). Por consiguiente, el submandato de idoneidad se satisface cuando se comprueba una relación empírica entre el medio escogido y el fin perseguido por la medida adoptada (Clérico, 2009, pp. 82 y 83).

El examen de necesidad, por su lado, exige una comparación entre medios; ello en miras a elección de aquel que resulte menos intrusivo. Es decir, supone la inexistencia de un recurso más moderado para conseguir el propósito perseguido con igual eficacia. En términos más sencillos: si hay dos medios disponibles e 
igualmente efectivos para promover un determinado principio, pero uno de ellos afecta de un modo menos intenso que el otro a un segundo principio, las posibilidades fácticas determinan la elección de aquel que importe un menor grado de afectación (Caminos, 2014, p. 56).

Finalmente, el contenido de la idea de proporcionalidad en sentido estricto es expresado mediante la ley de ponderación: "Cuanto mayor sea el grado de no satisfacción o restricción de un principio, tanto mayor debe ser la importancia de la satisfacción del otro" (Alexy, 1985, p. 161). Este punto, como ya se ha afirmado, reviste una importancia trascendental. Ello se debe a que, en tanto terreno propicio de la ponderación, permite establecer la relación de precedencia condicionada entre los principios en colisión y propiciar una solución al caso concreto, la que, además, podrá justificarse en términos de racionalidad.

De acuerdo con Alexy, la justificación racional de las decisiones adoptadas con base en la proporcionalidad en sentido estricto se logra a partir de tres elementos: La estructura de la ponderación, la fórmula del peso y el discurso racional (2006, pp. 7 a 10). Con relación al primero, sostiene que aquella puede dividirse en tres etapas: La primera, establece el grado de no satisfacción o restricción de un primer principio; la segunda, identifica el grado de importancia de satisfacción del principio opuesto; y, la última, determina si la importancia de satisfacer el segundo principio justifica el detrimento del primero. Esta estructura elemental posibilita la formulación de juicios racionales mediante la aplicación de la escala tríadica, esto es, la valoración de la satisfacción e intervención de los principios opuestos a partir de su identificación como leves, moderadas o graves. Así, por ejemplo, la afectación del derecho al honor que se originaría por publicitar información de relevancia pública referida a un funcionario en el ejercicio de su cargo podría catalogarse como leve o media, frente a la necesidad grave de satisfacer el derecho a la información en miras a ejercitar el control democrático de la gestión estatal. 
Sin perjuicio de lo antedicho, Alexy sostiene que la escala de tres intensidades es importante pero no suficiente para demostrar que la ponderación es racional. Para ello, es necesario dar un paso más. Ese paso más consiste en entender que la ponderación lleva implícito un sistema de inferencias que se consolida a través de la fórmula del peso, es decir, de una estructura ilativa de principios que se expresa mediante distintas variables (2006, p. 8). Ahora bien, la racionalidad de cualquier estructura inferencial está supeditada a la existencia de una conexión de premisas que puedan ser justificadas racionalmente. De este modo, afirma que las variables que conforman la fórmula del peso se expresan, en la práctica jurídica, en juicios y, estos -en tanto elementos del discurso- manifiestan una pretensión de corrección ${ }^{4}$ y arriban a una conclusión que debe ser justificada por medio de argumentos. De ello se desprende que la fórmula es una clase de argumento. Las formas de argumento definen la estructura lógica de movimientos dentro de los discursos y muestran cómo se relacionan entre sí. De allí se sigue la importancia asignada al discurso racional práctico, pues: la ponderación exige la formulación de argumentos

\footnotetext{
4 Para Robert Alexy (2009, pp. 81 y 82), el derecho tiene una doble naturaleza: comprende tanto una dimensión real como ideal. En la primera (dimensión real) se encuentran los elementos definitorios de la legalidad conforme al ordenamiento y de la eficacia social. En cambio, en la segunda (dimensión ideal) se encuentra la corrección moral. Es decir, existe una relación conceptual entre el Derecho y la Moral. Este punto reviste suma importancia porque permite entender la razón por la cual, para el autor, el discurso jurídico es un caso particular de discurso práctico general. Sobre este punto advierte que tanto en el discurso práctico general como en el discurso jurídico se efectúa una pretensión de corrección. La diferencia entre uno y otro radica en que, el primero se refiere a aquello que es correcto independientemente del Derecho positivamente válido en cada caso; en cambio, el segundo, se refiere a aquello que es correcto dentro del marco de un sistema jurídico determinado. Por consiguiente, la argumentación jurídica está sujeta a la ley y al precedente, y tiene que atender al sistema de Derecho elaborado por la dogmática jurídica. Es decir que, si la ley, el precedente y la dogmática prevén una solución para el caso, este debe resolverse adoptando la decisión que ellos proscriban. Pero, si estos no fijan respuesta alguna a la cuestión jurídica pendiente de resolución (casos difíciles) se ha de satisfacer la pretensión de corrección para efectuar valoraciones adicionales que no pueden extraerse del material autoritativo preestablecido. Para efectuar la mentada satisfacción, esas premisas adicionales tienen que justificarse por medio de la argumentación práctica general que incluye al discurso moral.
} 
susceptibles de justificar la elección de una determinada medida; esos argumentos se expresan en juicios; y, precisamente, los juicios son elementos esenciales del discurso.

En este punto, es necesario advertir que en el razonamiento jurídico no se puede esperar la elaboración de un método que nos permita llegar a una solución definitiva para cada caso difícil que se plantee; en otras palabras, en las ciencias sociales no hay conocimientos concluyentes, es decir, que excluyan la falsedad o garanticen la verdad. Incluso Alexy reconoce que la ponderación no prescribe resultados definitivos, sino que “(..) nos dice cómo argumentar cuando solo se puede cumplir con un principio a costa de otro" (Alexy, 1994, p. 48) y que "la teoría del discurso no es una maquinaria que nos permita determinar exacta, objetiva y definitivamente el peso de cada derecho" (Alexy, 1994, p. 49). No obstante, sí se puede aspirar a la creación de estructuras formales que ordenen el razonamiento y la consiguiente argumentación. ${ }^{5}$ Eso se debe a que la imposibilidad de brindar certezas no debe confundirse con la inexistencia de buenos fundamentos para defender la adopción de una determinada decisión. Creo que el valor del principio de proporcionalidad está en línea con el mentado anhelo porque permite identificar los pasos argumentativos que han de seguirse cuando la decisión versa sobre la restricción de un derecho en miras a la protección de otro y, a su vez, facilita la actividad de contralor sobre el producto resultante - en este caso, de la conducta desplegada por la Administración pública-.

${ }^{5}$ Sobre la racionalidad de la ponderación en tanto estructura formal véase Mocoroa, 2017, pp. 73-85. 


\section{El derecho de acceso a la información pública}

\section{a. Caracterización}

Cuando se analiza el derecho de acceso a la información pública - esto es, la facultad que se le reconoce a toda persona para solicitar y recibir información completa, veraz, adecuada y oportuna ${ }^{6}$-, deben tenerse en cuenta algunas cuestiones relevantes. La primera consiste en reconocer que éste puede ser abordado desde una doble perspectiva: por un lado, en su dimensión individual - como un derecho fundamental oponible al Estado-; y, por el otro, en su dimensión institucional —como la obligación estatal de adecuar su estructura interna para garantizarlo- - A decir verdad, se trata de un derecho humano derivado de la libertad de pensamiento y de expresión, ${ }^{7}$ estrechamente vinculado a la democracia ${ }^{8} \mathrm{y}$ al principio republicano de gobierno ${ }^{9}$ que, correlativamente, consagra un deber

\footnotetext{
${ }^{6}$ Ley $\mathrm{N}^{\circ} 104$, Artículo $1^{\circ}$, Derecho de acceso a la información pública: “Toda persona tiene derecho a solicitar y a recibir información completa, veraz, adecuada y oportuna. Para ejercer el derecho de acceso a la información pública no será necesario acreditar derecho subjetivo, interés legítimo o razones que motiven la petición.
}

Implicará la libertad de acceder, solicitar, recibir, copiar, analizar, reprocesar y redistribuir información bajo custodia de los sujetos obligados, con las únicas limitaciones y excepciones que establece la presente ley".

${ }^{7}$ La mentada derivación ha sido reconocida por la Corte Interamericana de Derechos Humanos en el caso Claude Reyes y otros vs. Chile en el cual el tribunal convencional condenó al Estado chileno.

${ }^{8}$ Sobre este punto, se advierte que la CSJN ha llegado a calificar a la información como el "oxígeno de la democracia" (CSJN, Asociación por los derechos civiles c/ PAMI, Fallos: 335:2393, 2012).

${ }_{9}$ El sistema republicano de gobierno se encuentra consagrado en el primer artículo, tanto de la Constitución Nacional (La Nación Argentina adopta para su gobierno la forma representativa republicana federal...), como de la Constitución de la Ciudad Autónoma de Buenos Aires (La Ciudad de Buenos Aires [...] adopta para su gobierno la forma republicana y representativa...). El hecho de que sea lo primero que nuestras constituciones receptan, da cuenta de su gran importancia institucional.

El republicanismo presenta ciertas notas características, entre las que se destacan las siguientes: la división de poderes; el sistema de frenos y contrapesos; $y$, el principio de publicidad de los actos de gobierno. Este último tiene por finalidad garantizar a los 
en cabeza del Estado consistente en dar publicidad a aquella información que revista interés público. ${ }^{10}$ De esta primera consideración se deriva, como consecuencia lógica, la segunda: es decir, el alcance limitado de las excepciones a la publicidad. Eso se debe a que, dada la importancia institucional asignada al derecho en análisis — por cuanto posibilita que cualquier persona conozca la manera en que sus funcionarios y gobernantes se desempeñan-, es razonable que cualquier límite que se intente contra él deba interpretarse restrictivamente (principio de máxima divulgación). ${ }^{11}$ En consonancia con lo expuesto, es preciso mencionar que, en esta materia, se invierte la carga de la prueba ya que es el Estado el que debe demostrar, en cada caso, que la restricción cumple con ciertos parámetros suficientes para justificarla.

De lo antedicho se desprende que no obstante las notables características del derecho de acceso a la información, éste no es absoluto. Sin embargo, para que las restricciones que se invoquen contra él estén legitimadas, es necesario cumplir con una serie de requisitos previos. Siguiendo esta línea, se han elaborado algunos criterios de ponderación — como el test del daño y el test del interés público $^{12}$ - y otras tantas pautas argumentativas, cuya observancia es

representados el conocimiento acerca de la forma en la que sus representantes gobiernan. En este punto, la relación entre el republicanismo y el derecho de acceso a la información pública es clara; pues, la publicidad de los actos de gobierno requiere, invariablemente, de un sistema eficiente de acceso a la información pública, cuyo fundamento no es otro que el ejercicio del control democrático de la gestión estatal.

${ }^{10}$ La doctrina tiene dicho que "el derecho a la información pública (...) es un derecho bidireccional que incluye tanto el derecho a informar, cuyo titular es el sujeto activo, y el derecho a ser informado, siendo el titular el sujeto pasivo en la relación" (Basterra, 2006, p. 299).

${ }^{11}$ Es importante destacar que la Corte IDH sostuvo que "en una sociedad democrática es indispensable que las autoridades estatales se rijan por el principio de máxima divulgación, el cual establece la presunción de que toda información es accesible, sujeto a un sistema restringido de restricciones" (Corte IDH, Claude Reyes y otros vs. Chile, Serie C. № 151, 2006, párr. 92).

12 Tanto el test del daño como el del interés público (ambos consagrados en la Ley Modelo Interamericana sobre Acceso a la Información Pública y receptados por el 
condición determinante para tener por "fundada" una denegatoria de información.

Los criterios de ponderación mencionados en el párrafo anterior se inspiraron en los lineamientos sentados por la Corte IDH en el caso "Claude Reyes y otros vs. Chile". Allí se estableció que: "las restricciones que se impongan [al derecho de acceso a la información] deben ser necesarias en una sociedad democrática, lo que depende de que estén orientadas a satisfacer un interés público imperativo. Entre las varias opciones para alcanzar ese logro, debe escogerse aquella que restrinja en menor escala el derecho protegido. Es decir, la restricción debe ser proporcional al interés que la justifica y debe ser conducente para alcanzar el logro de ese legítimo objetivo, interfiriendo en la menor medida posible en el efectivo ejercicio del derecho". ${ }^{1314}$ Asimismo, se ha aseverado que aquellas deben estar previamente fijadas en la ley. ${ }^{15}$

Como puede apreciarse, el tribunal convencional no exige otra cosa que una argumentación basada en la aplicación del principio de proporcionalidad. Por esa razón, en el siguiente apartado, se

\footnotetext{
Órgano Garante del Derecho de Acceso a la Información Pública en la CABA) suponen un mecanismo de "balanceo" entre dos derechos opuestos. Dicho mecanismo cuenta con tres etapas: En la primera, se establece el grado de insatisfacción del acceso a la información. En la segunda, se determina el grado de satisfacción del bien jurídico protegido por la excepción invocada por el sujeto obligado al momento de denegar una solicitud de información. Finalmente, en la tercera etapa, se establece si la importancia de satisfacer la seguridad pública justifica la insatisfacción del acceso a la información. Ambos test aspiran a excluir la discrecionalidad del sujeto obligado por medio de la elaboración de argumentos con peso suficiente que justifiquen la procedencia de la excepción invocada. Sin embargo, el primero (test del daño) suele aplicarse en los casos en que el interés comprometido es predominantemente público (i.e. protección de la seguridad pública); mientras que el segundo tiene lugar en los casos en que los bienes afectados con relevancia pública son los derechos de las personas (i.e. protección de datos sensibles).

${ }^{13}$ Corte IDH, Claude Reyes y otros vs. Chile, Serie C. № 151, párr. 91, 2006.

14 CSJN, Garrido, Fallos 339: 827, considerando 5, 2016.

${ }^{15}$ Corte IDH, Claude Reyes y otros vs. Chile, Serie C. № 151, párr. 89, 2006.
} 
procurará determinar su operatividad en este supuesto específico de colisión entre derechos.

\section{b. El principio de proporcionalidad en el derecho de acceso a la información pública}

Teniendo en cuenta las pautas delineadas por la jurisprudencia y la doctrina, puede decirse que la procedencia de las excepciones a la publicidad está supeditada al cumplimiento de dos principios: el de legalidad y el de proporcionalidad en sentido estricto. El primero exige que toda limitación que se intente contra el derecho de acceso a la información esté previamente definida en forma precisa y clara a través de una ley formal y material. ${ }^{16}$ Tal es así que, desde el punto de vista del sujeto obligado, se cumple con dicha manda transcribiendo el texto de la ley que consagra la excepción invocada como motivo de la denegatoria. Sin perjuicio, la transcripción pura y simple de la disposición respectiva no es suficiente para calificar a esta última como "fundada". ${ }^{17}$ Por eso, una vez cumplido con el principio de

16 Ídem. Asimismo, Informe $\mathrm{N}^{\circ} 14196076 / \mathrm{OGDAI} / 2018$ adjunto a la Resolución $\mathrm{N}^{\circ}$ 6/OGDAI/2018.

${ }^{17}$ Esta aclaración cobra especial relevancia si se tiene en cuenta que, en ciertos casos, los sujetos obligados han denegado solicitudes de información limitándose a invocar el texto de la ley. Es decir, sin fundamentar la razón por la que consideran que lo pedido debe mantenerse reservado. Un ejemplo claro de esta cuestión se observa en aquellas solicitudes que tienen por objeto acceder a información vinculada a las vacantes de escuelas. Allí, en lugar de fundamentar la denegatoria, el sujeto obligado se limitó a mencionar el artículo 6 inciso c) de la Ley $\mathrm{N}^{\circ} 104$ que protege aquella información cuya publicidad pudiera revelar la estrategia a adoptarse en la defensa o tramitación de una causa judicial en la cual es sujeto obligado sea parte, o divulgare las técnicas o procedimientos de investigación y a remitir al solicitante a un proceso colectivo bajo el argumento de que los datos requeridos en el pedido de acceso se habrían aportado allí. $\mathrm{Al}$ respecto, véase Juzgado de Primera Instancia en lo Contencioso Administrativo y Tributario $\mathrm{N}^{\circ} 11$, Secretaría $\mathrm{N}^{\circ}$ 22, Barreyro, Eduardo Daniel c/ GCBA s/ acceso a la información (incluye Ley 104 y Ambiental), Expte. $\mathrm{N}^{\circ}$ 1139/2019-0, 2019; Juzgado de Primera Instancia en lo Contencioso Administrativo y Tributario $\mathrm{N}^{\circ} 21$, Secretaría $\mathrm{N}^{\circ}$ 42, Barreyro, Eduardo Daniel c/ GCBA s/ acceso a la información (incluye Ley 104 y Ambiental), Expte. $\mathrm{N}^{\circ} 1146 / 2019-0,2019$; Juzgado de Primera Instancia en lo Contencioso Administrativo y Tributario $\mathrm{N}^{\circ} 12$, Secretaría $\mathrm{N}^{\circ} 24$, Barreyro, Eduardo 
legalidad, se deberán satisfacer los presupuestos derivados de la aplicación del principio de proporcionalidad.

En efecto, el examen acerca de la "idoneidad" de la medida supone que el sujeto obligado se pregunte lo siguiente: ¿̨cuál es el fin para reservar la información y su fundamento? ¿̨la reserva posibilita la consecución de aquel? ${ }^{18}$ La respuesta a ellos requiere no solo la identificación de una colisión entre principios -con especial atención a aquel que protege la excepción- sino, además, la demostración de que la limitación sirve para fomentar la realización o el resguardo del principio opuesto al acceso a la información.

Por su lado, para que la restricción sea "necesaria" debe acreditarse que no existen medios alternativos menos gravosos para proteger el principio amparado por la excepción. Es decir, ise puede evitar la restricción del derecho de acceso a la información pública a través de otro medio, o por lo menos, reducir el grado de limitación? La respuesta a este interrogante presupone una "comparación" entre medios que constituye el núcleo del presupuesto en análisis (Clérico, 2009, p. 101).

En el ámbito del derecho de acceso a la información pública, la comparación entre dos medios aptos para fomentar la protección de un principio en miras a la elección de aquel que restrinja en menor medida el derecho contrapuesto se produce al evaluar la posibilidad de disociación. De este modo, cuando el sujeto obligado invoca una excepción legal cuenta con dos opciones: la denegatoria total o parcial de los datos requeridos. La diferencia entre ambas radica en que, en la primera, no se entrega la información y, en la segunda, se entrega

\footnotetext{
Daniel c/ GCBA s/ acceso a la información (incluye Ley 104 y Ambiental), Expte. $\mathrm{N}^{\circ}$ 1149/2019- 0, 2019; entre otros.

${ }_{18}$ Informe $\mathrm{N}^{\circ}$ 14196076/OGDAI/2018 adjunto a la Resolución $\mathrm{N}^{\circ}$ 6/OGDAI/2018 y Resolución $\mathrm{N}^{\circ}$ 51/OGDAI/2018.
} 
solo una parte de ella (es decir, se facilita la porción sujeta a publicidad y se reserva la que está protegida por la excepción). ${ }^{19}$

La aplicación estricta del examen del medio alternativo menos lesivo determina que si la disociación es posible, el sujeto obligado debe aplicarla; esto es, entregar la información solicitada y ocultar aquella que esté sujeta a reserva mediante el empleo de algún mecanismo apto para ello (v.g. sistema de tachas). En este caso debe, asimismo, indicar qué parte del documento se ha reservado, la fuente de la información y la razón por la cual se considera aplicable la excepción respectiva (cf. Art. 7, Decreto $N^{\circ}$ 260/17).

Sin perjuicio de lo expuesto, puede suceder que la "separación" de la información requerida sea imposible. Es decir que ella, en su totalidad, esté protegida por la excepción invocada como motivo de la reserva. En estos casos, la restricción al derecho de acceso a la información es inevitable; pues, el único medio disponible para proteger un principio consiste en invocar la excepción que lo resguarda y, en consecuencia, denegar la solicitud de información.

Desde este punto de vista, la ineludible afectación que el derecho de acceso a la información debe "soportar" para proteger un principio opuesto exige que el sujeto obligado de buenas razones para justificarla. En consecuencia, se cumple con la pauta aquí examinada siempre que, al momento de fundar la denegatoria, se brinden

\footnotetext{
${ }^{19} \mathrm{El}$ artículo $7^{\circ}$ de la Ley $\mathrm{N}^{\circ} 104$ establece que "[e]n caso que exista un documento que contenga en forma parcial información cuyo acceso esté limitado en los términos del Artículo $6^{\circ}$, debe suministrarse el resto de la información solicitada". En consonancia con lo expuesto, el Decreto $\mathrm{N}^{\circ} 260 / 17$-reglamentario de la Ley $\mathrm{N}^{\circ} 104$ - establece que: "[c]uando un documento contenga información cuyo acceso esté limitado en los términos del artículo $6^{\circ}$ de la ley, deberá suministrarse el resto de la información utilizando el sistema de disociación. La información no comprendida deberá ser entregada o publicada en una versión del documento que tache, oculte o disocie aquellas partes sujetas a la excepción y se indicará qué parte del documento ha sido mantenida bajo reserva, la fuente de la información y su encuadre legítimo en alguna de las hipótesis de excepción previstas en la Ley $\mathrm{N}^{\circ} 104 "$.
} 
razones normativas por las cuales resulta imposible inclinarse por la herramienta disociativa.

Por último, la excepción debe ser analizada a la luz de la proporcionalidad en sentido estricto; es decir, el sujeto obligado debe acreditar que el riesgo al principio fomentado mediante la denegatoria de la información supera el interés de difundirla. Para ello, no basta con calificar el grado de intensidad de la restricción y de la satisfacción de los principios opuestos y compararlos a efectos de determinar cuál pesa más, ni tampoco apelar a la producción de daños hipotéticos o conjeturales para denegar la información. Por el contrario, la interpretación restrictiva que rige en la materia plantea la necesidad de demostrar el riesgo real y específico que produciría la publicidad de los datos solicitados en el caso concreto. En otras palabras, las determinaciones de tipo cuantitativo que proporciona la escala triádica son importantes, pero no suficientes ya que las circunstancias vigentes exigen que, además de establecer una relación de precedencia condicionada entre principios opuestos, se exhiban las razones por las cuales en ese caso en particular se adopta la decisión respectiva. Para ello, puede apelarse a decisiones pasadas en las que se haya resuelto de ese modo. Por ejemplo, si la solicitud de información tiene por objeto conocer si un funcionario público y un ciudadano común han recibido una vacuna contra el COVID-19 en el contexto de revuelo por la llamada "vacunación VIP”, el Estado podría apelar al estándar sentado por la CSJN en "Ponzetti de Balbín” ${ }^{20}$ a efectos de dar publicidad al primer supuesto, pero denegar la información en el segundo. En efecto, no bastaría que para proceder de tal forma se dijera que el trato diferenciado obedece a que, en el primer caso, existe un interés público imperativo (y por lo tanto "grave") en conocer la información que postula su precedencia respecto del derecho a la intimidad que sufre un grado de restricción "leve" o "medio", sino que es necesario que se justifique ese proceder. Así, se podría aseverar que como los funcionarios públicos tienen una

20 CSJN, Ponzetti de Balbín, Fallos 306:1892, considerando 9, 1984. 
menor expectativa de privacidad en todo aquello que concierne al ejercicio de su actividad, la circunstancia demanda que esa información sea divulgada; circunstancia que no se presenta en nuestro segundo supuesto. En efecto, este último se refiere a un ciudadano común, razón por la cual aquí ha de prevalecer el derecho a la intimidad, es decir, la confidencialidad de los datos referidos a la salud que, además, integran la historia clínica del paciente y, por lo tanto, son reservados.

En síntesis, el cumplimiento de los tres últimos requisitos analizados exige que el sujeto obligado adopte una decisión estrictamente fundamentada que permita conocer los motivos por los cuales: invoca una excepción legal para denegar una solicitud de información; aplica o no mecanismos de disociación; y, finalmente, coloca al principio protegido como un valor que, en el caso concreto, precede a la publicidad de la información.

Por último, me gustaría advertir que no escapa de mi conocimiento que un esquema de este tipo, pese a resultar seductor en la teoría, tiende a presentar complicaciones en la práctica, ya sea por desconocimiento técnico o falta de tiempo. Sin embargo, creo que, como todo ideal, la estructura aquí presentada ha de servir para inspirar la redacción de argumentos serios, sólidos y respetables que, en última instancia, hagan cierta la legitimidad de la medida adoptada.

\section{Conclusión}

En este trabajo se intentó demostrar que en tanto la validez de una decisión restrictiva de un derecho fundamental está supeditada a la presentación de buenas razones para su adopción, el principio de proporcionalidad se erige como una herramienta apropiada para tal finalidad. En efecto, la estructura argumentativa propuesta por él posibilita que cualquier persona pueda controlar y, eventualmente, criticar la actividad desplegada por el gobierno —específicamente 
cuando se trata de restringir el acceso a la información pública en miras a la protección de un bien jurídico consagrado legalmente como una excepción-. Sin perjuicio de lo expuesto, se señaló que las justificaciones de tipo cualitativo exigidas por la escala tríadica no son suficientes para tener por fundada la restricción ya que, en última instancia, el peso asignado a cada principio en pugna depende de los elementos tomados en cuenta por quien efectúa la limitación. A las consideraciones de grado, entonces, se han de sumar las justificaciones de las variables presentadas en la fórmula del peso; puesto que solo de este modo se podrá dar cuenta de las razones que han sido consideradas para determinar por qué la prioridad del principio por medio del cual se deniega la publicidad de la información se justifica en el caso concreto. En otras palabras, no basta con señalar que la intensidad de la satisfacción de un primer principio legitima la restricción de un segundo principio, sino que es preciso que se emitan juicios por medio de los cuales se den buenas razones; esto es, motivos convincentes para aceptar la razonabilidad de la medida limitativa. De este modo, si bien no se elimina la discrecionalidad de quien la dispone, se hace explícita la justificación normativa subyacente a ella; lo que permite disociar aquellos razonamientos jurídicos serios de los que no lo son. En efecto, solo este tipo de fundamentación deja a salvo el basamento sobre el cual se estructura el derecho de acceso a la información —aún cuando éste se restringe-, a saber: el control ciudadano sobre la legitimidad o ilegitimidad de los asuntos públicos.

\section{Referencias}

Libros y artículos de doctrina

Alexy, R. (1985), Theorie der grundrechte (Teoría de los derechos fundamentales), Madrid: Centro de Estudios Constitucionales.

Alexy, R. (1994), "Derechos, razonamiento jurídico y discurso racional", ISONOMÍA, $\mathrm{N}^{\circ} 1$, pp. 37 a 49. 
Alexy, R. (2006), “Ponderación, control de constitucionalidad y representación”, M.C., J.O.H, y R.V., Jueces y ponderación, México: Universidad Nacional Autónoma de México, pp. 1 a 18.

Alexy, R. (2008), "La fórmula del peso”, M.C., El principio de proporcionalidad y la interpretación constitucional, Quito: Ministerio de Justicia y Derechos Humanos, pp. 13 a 42.

Alexy, R. (2009), "Los principales elementos de mi filosofía del derecho", $D O X A, \mathrm{~N}^{\circ} 32$, pp. 67 a 84 , https://doi.org/10.14198/DOXA2009.32.04.

Basterra, M., Ciudad transparente: Ley $n^{\circ} 104$ comentada, Ley $n^{\circ}$ 5784 y decreto reglamentario $n^{\circ} 260 / 17$, 1a ed., Argentina: Editorial Jusbaires, 2018.

Caminos, P.. "El principio de proporcionalidad: ¿ Una nueva garantía de los derechos constitucionales?", Revista Electrónica del Instituto de Investigaciones “Ambrosio L. Gioja”, 2014.

Clérico, L. (2009), El examen de proporcionalidad en el derecho constitucional, Argentina: Eudeba.

Mocoroa, J.M., “La racionalidad de la ponderación en la argumentación constitucional”, Revista Prolegómenos Derechos y valores, 2017, pp. 73-85.

Jurisprudencia

Corte IDH, Claude Reyes y otros vs. Chile, Serie C. № 151, 2006.

CSJN, Ponzetti de Balbín, Fallos 306:1892, 1984.

CSJN, Asociación por los derechos civiles c/ PAMI, Fallos: 335:2393, 2012.

CSJN, Garrido, Fallos 339: 827, 2016.

Juzgado de Primera Instancia en lo Contencioso Administrativo y Tributario $\mathrm{N}^{\circ} 11$, Secretaría $\mathrm{N}^{\circ} 22$, Barreyro, Eduardo Daniel c/ GCBA s/ acceso a la información (incluye Ley 104 y Ambiental), Expte. $\mathrm{N}^{\circ} 1139 / 2019-0,2019$. 
Juzgado de Primera Instancia en lo Contencioso Administrativo y Tributario $\mathrm{N}^{\circ} 21$, Secretaría $\mathrm{N}^{\circ} 42$, Barreyro, Eduardo Daniel c/ GCBA s/ acceso a la información (incluye Ley 104 y Ambiental), Expte. $\mathrm{N}^{\circ}$ 1146/2019-0, 2019.

Juzgado de Primera Instancia en lo Contencioso Administrativo y Tributario No 12 - Secretaría No 24, Barreyro, Eduardo Daniel c/ GCBA s/ acceso a la información (incluye Ley 104 y Ambiental), Expte. $\mathrm{N}^{\circ}$ 1149/2019- 0, 2019. 